\title{
Planificación del mantenimiento en las universidades públicas de la Costa Oriental del Lago
}

\author{
Maintenance planning in public universities on the East Coast of Lake
}

Daryeling Betancourt

daryeling@gmail.com

Código ORCID: 0000-0002-1558-9650

Universidad del Zulia, Núcleo Costa Oriental del Lago. Venezuela

La investigación tuvo como propósito describir la planificación del mantenimiento en las universidades públicas de la Costa Oriental del Lago. El tipo de investigación fue descriptivo con diseño no experimental, transeccional y de campo. La población quedó constituida por las personas que se desempeñan en el área de mantenimiento de las universidades analizadas. La técnica para la recolección de datos fue la encuesta, siendo el instrumento un cuestionario conformado por 12 ítems con cinco alternativas de respuestas, el mismo fue validado por expertos, mientras que su confiabilidad se determinó a través del coeficiente de Alpha Cronbach, obteniéndose 0,932 . La técnica de análisis utilizada fue a través de la media aritmética. Se concluye que estas organizaciones otorgan alta presencia del plan estratégico, implementación del plan estratégico, ejecución del plan estratégico, así como de la evaluación y control del plan estratégico, en su gestión de mantenimiento, lo cual les atribuye una alta fortaleza.

Palabras clave: ejecución del plan estratégico, evaluación y control del plan estratégico, gestión de mantenimiento, implementación del plan estratégico, plan estratégico, planificación del mantenimiento

AbstracT

The purpose of the research was to describe the maintenance planning in the public universities of the Eastern Lake Coast. The type of research was descriptive with a non-experimental, transectional, field design. The population was made up of people who work in the maintenance area of the analyzed universities. The technique for data collection was the survey, the instrument being a questionnaire made up of 12 items with five alternative responses, it was validated by experts, while its reliability was determined using the Alpha Cronbach coefficient, obtaining 0.932. The analysis technique used was through the arithmetic mean. It is concluded that these organizations grant high presence of the strategic plan, implementation of the strategic plan, execution of the strategic plan, as well as the evaluation and control of the strategic plan, in their maintenance management, which attributes a high strength to them.

Keywords: execution of the strategic plan, evaluation and control of the strategic plan, maintenance management, implementation of the strategic plan, strategic plan, maintenance blannina 


\section{INTRODUCCIÓN}

En ocasiones, se mira la gestión de mantenimiento como una operación que tiene por finalidad orientar los esfuerzos para evitar fallas en los equipos de producción. Sin embargo, no se ve que esta gestión es realmente estratégica, sobre todo en el sector de educación superior, donde la capacidad productiva debe maximizarse y el mantenimiento debe ser función directa de la confiabilidad operacional. Todo esto para lograr que los equipos operen no sólo con una elevada confiabilidad sino también dentro de sus parámetros de diseño con el fin de disponer de procesos productivos óptimos.

Desde esta perspectiva, Arata (2009) afirma que, el concepto actual de gestión de mantenimiento no está enfocado a la reparación de un equipo en el menor tiempo posible, sino más bien en mantenerlos en operación a los niveles especificados. Es por esto, que los cambios y requerimientos implican directamente a las unidades de ingeniería y mantenimiento de todas las empresas en general, quienes tienen bajo su responsabilidad garantizar la disponibilidad de los recursos físicos y tecnológicos.

De manera que, hoy día, las empresas competitivas se adecuan a una gestión de mantenimiento que hace uso de sus recursos económicos, materiales y de tiempo de una manera más efectiva y eficiente, conllevando a la mejora continua de los procesos. Sin embargo, Amendola (2006) afirma que, existen empresas que se encuentran saturadas de una gran cantidad de métodos de mantenimiento que se usan indiscriminadamente, por el deseo de no quedarse atrás y estar dentro de las corrientes de pensamientos actuales, utilizándolas como fin y no como medio para alcanzar excelencia.

Aunado a esto, agrega Amendola (2006), aún existen empresas que se apoyan en viejos modelos de mantenimiento, otras que ni siquiera poseen una dirección sólida de gestión, derivándose fracasos como efecto de inapropiadas decisiones. Todo esto por el afán de obtener beneficios inmediatos, se toman caminos equivocados generando peores condiciones de las que presentaba la organización, esto implica para el mantenimiento, retos y oportunidades que merecen ser valorados.

En relación con esto, Alpizar (2005) opina que, la gestión de mantenimiento debe orientarse hacia el desarrollo de actividades con el fin de conservar las instalaciones de los equipos en condiciones de funcionamiento seguro, eficiente y económico. Para ejecutar una aplicación efectiva del mantenimiento, afirma este autor, se debe realizar una base de datos con la información general de las maquinarias $y$, posteriormente, hacer un plan de inspección oportuno que sólo puede aplicarse al tener los datos del equipo. Además, agrega el autor, el mantenimiento de equipos representa una inversión a mediano y largo plazo, debido a que generará ganancias no sólo para la empresa a quién esta inversión se le revertirá en mejoras en su producción, sino también en la disminución de índice de accidentalidad laboral, logrando así mejorar los procesos organizacionales.

En la actualidad, se observan cambios en cuanto a los nuevos métodos como técnicas de mantenimiento, contando con diversas actividades. La idea del mantenimiento está cambiando, debido a un aumento en los procesos, mayor complejidad de los equipos, nuevas técnicas de mantenimiento, así como, un nuevo enfoque de la organización, colocando para ello a prueba los limites, las actitudes y conocimiento del personal en toda la rama de la industria, donde el personal de mantenimiento desde el artesano al gerente tiene que adoptar nuevas formas de pensar actuando.

Es por ello, que la gestión se ha modificado en el tiempo para dar respuesta a la extraordinaria complejidad de los sistemas organizativos que las empresas han venido adoptando. Autores como Arata (2009) y Duffuaa 
y otros (2010), afirman que la evolución de las técnicas de mantenimiento ha ido siempre en concordancia con las evoluciones tecnológicas, que permiten incrementar significativamente el aprendizaje acerca del conocimiento degenerativo interno de los equipos, que hace tan solo unos cuantos años era prácticamente desconocido.

Por tanto, se hace necesario contar con equipos en óptimas condiciones en todo momento, para asegurar una disponibilidad total del sistema, lo cual está basado en carencia de errores y fallas. El mantenimiento debe procurar un desempeño continuo y operar bajo las mejores condiciones técnicas, sin importar las condiciones externas del ambiente, además debe estar destinado a optimizar la producción, reducir los costos por averías y disminuir gastos.

Aun cuando, a nivel mundial, estas empresas han venido superando obstáculos, un gran número de gerentes, siguen sin conocer los patrones esenciales para optimizar sus operaciones. Así pues, para Nava (2006), el mantenimiento es importante ya que permite mejorar los niveles de producción de las empresas, bien sea manufactureras $o$ de servicios. Al planificar un buen programa de mantenimiento de los equipos, se logra mejorar los recursos que se ven reflejados en la utilidad de la empresa.

Es así como adoptar o implementar un adecuado plan de mantenimiento permite lograr mayores niveles de confiabilidad, mantenibilidad, disponibilidad y rendimiento de los equipos dando, como beneficio final la disminución del índice de fallas pudiendo cumplir con la producción deseada al menor costo posible que brinden mejores ventajas a la organización, sobre todo dentro del desarrollo de sus actividades de mantenimiento.

Ahora bien, en el caso que compete a la investigación, cabe reseñar que, en Venezuela, las universidades públicas, orientándose según los valores presentes en las filosofías de gestión que las sustentan, deben justificar su espacio en la sociedad a la que atienden, dando cuenta de la inversión/gasto que representan en relación directa con los resultados/beneficios que producen (Zambrano, 2008). De allí que a través de una adecuada planificación del mantenimiento, se minimicen los costos por el deterioro de las instalaciones, transporte $y$ equipos, lo que conllevaría a mejorar la rentabilidad de estas instituciones.

Finalmente, con base a las consideraciones presentadas en los párrafos anteriores, y tomando en cuenta que las organizaciones en general y las universidades públicas de la Costa Oriental del Lago en lo particular, deben dar respuesta satisfactoria a sus clientes, mediante una adecuada gestión que incluya el mantenimiento como parte garante de la eficiencia operacional, se realizó esta investigación, con el propósito de describir la planificación del mantenimiento en las universidades públicas de la Costa Oriental del Lago.

\section{Gestión de mantenimiento}

Para Rodríguez (2008), la gestión de mantenimiento surge como todas aquellas actividades de diseño, planificación y control destinadas a minimizar todos los costos asociados al mal funcionamiento de los equipos. Entre éstas se incluyen, además de las funciones típicamente asociadas al mantenimiento, los estudios de la posibilidad de renovación de equipos, la realización de modificaciones que ayuden aumentar la confiabilidad y flexibilizar el funcionamiento, la formación del personal de producción para la realización de funciones pequeños mantenimientos.

En este marco de referencia, la gestión del mantenimiento es concebida por Zambrano y Leal (2006) como, un proceso sistémico donde a través de una serie de medidas organizativas se pueden planear las acciones de las actividades de mantenimiento por medio de procedimientos que lleven un orden o secuencia lógica de esta función, a fin de conseguir un constante y adecuado desempeño de los 
equipos pertenecientes al sistema productivo, esto con la finalidad de identificar los pasos a seguir y prever las posibles desviaciones que se puedan presentar durante el desarrollo de estas actividades de mantenimiento.

Por su parte, Kelly (2006) aclara que, la gestión del mantenimiento está referida a la efectiva y eficiente utilización de los recursos materiales, económicos, humanos y de tiempo para alcanzar los objetivos de mantenimiento. Asimismo, Matalobos (2003) considera que, son aquellas actividades necesarias y orientadas a preservar los diferentes sistemas, para cumplir con el servicio que prestan en concordancia a un parámetro definido de estado de operación normal, contribuyendo de esta forma a conservar las actividades productivas derivadas de éstos.

Retomando los aportes de Zambrano y Leal (2006), quienes muestran un modelo de gestión de mantenimiento basado en la teoría administrativa, teoría organizacional, ciclo de calidad total y filosofía del mantenimiento de clase mundial, el cual se fundamenta en seis fases, siendo éstas: planificación, programación, seguimiento, ejecución, control y evaluación; contando, además, de 36 procedimientos que deberían realizarse para que la función mantenimiento de cualquier empresa tienda a ser lo más efectiva posible.

Adicionalmente, el modelo propuesto permite la retroalimentación o evaluación continua de la función mantenimiento, ya que a partir de esto se pueden diseñar medidas para el mejoramiento continuo, además ayuda a optimizar los recursos con los que cuenta la empresa, y lo más importante, tiende a optimizar costos por paradas no programadas, factor que afecta en gran medida la productividad de cualquier sistema productivo.

Es preciso destacar que el modelo de gestión de mantenimiento planteado por Leal y Zambrano (2006), es similar a lo establecido en la Norma Venezolana COVENIN 2500 (1993): "Manual para evaluar los sistemas de mantenimiento en la industria", indicando seis (6) áreas susceptibles al momento de evaluar la gestión, siendo estas: organización de la empresa, organización del mantenimiento, planificación de mantenimiento, mantenimiento: rutinario, programado, correctivo, preventivo, o por avería, personal de mantenimiento y apoyo logístico y recursos; los cuales fueron considerados en esta investigación.

Complementando lo antes expuesto, Duffuaa y otros (2010) exponen que, para gestionar de forma eficiente el mantenimiento es necesario que este sea visto como un sistema, ya que en el intervienen muchos factores que pueden influir en su éxito, no puede ser visto de manera aislada. Los gerentes deben visualizar el mantenimiento como un sistema para así mejorar el proceso, optimizando recursos, garantizando equipos confiables con pocas fallas que no causen interrupciones en el proceso productivo.

Asimismo, consideran que un sistema de mantenimiento puede verse como un modelo sencillo de entrada-salida. Las entradas son mano de obra, equipos, administración, herramientas, entre otros; y la salida sería equipos funcionando de manera confiable y bien configurado para lograr la operación planeada. Esto permite optimizar los recursos para aumentar al máximo las salidas de un sistema de mantenimiento.

A partir de los criterios formulados por los autores citados, en relación al concepto de gestión de mantenimiento, la investigadora comprende que, esta gestión se refiere al conjunto de acciones $\circ$ actividades que se realizan a un sistema, equipo o componente para asegurar que continúe desempeñando las funciones deseadas dentro de un contexto operacional determinado. Es decir, constituye un elemento importante para el sistema productivo de toda organización tomando en cuenta aspecto como capital, trabajo, técnicas, motivación, control y planificación, entre otros. 


\section{Planificación del mantenimiento}

Según Dounce (2011), planificar consiste en fijar el curso concreto de acción a seguir, estableciendo los principios que lo orientarán, secuencia de operaciones para realizarlo junto a la determinación del tiempo necesario para su realización. Por lo tanto, para planificar se debe definir, antes que todo, el objetivo, o sea lo que se quiere obtener con las acciones. Luego, se derivarán de éste las políticas, enunciados que marcan guías para facilitar las labores gerenciales; de seguido se determinará el procedimiento que se ha de seguir, con lo cual se puede definir la interrelación de recursos por emplear, incluyendo el tiempo, lo que proporciona los programas.

De forma más específica, para Koontz y Weihrich (2008), la planificación es un proceso que comienza por definir los objetivos, estrategias, políticas, procedimientos y planes detallados para alcanzarlos, establece una organización para la instrumentación de las decisiones e incluye una revisión del desempeño y mecanismo de retroalimentación para el inicio de un nuevo ciclo de implantación.

Ahora bien, en el ámbito de la gestión del mantenimiento, Jiménez y Milano (2006) afirman que, el proceso de planificación está dirigido a producir un determinado estado futuro al cual se desea llegar, que no se puede conseguir a menos que, se emprendan las acciones precisas y adecuadas.

Por lo tanto, planificar exigiría: que se tomen decretos anticipadamente, determinando lo que se hará, así cómo se hará antes de que llegue el momento de la ejecución, un cabal conocimiento de la organización o unidad responsable, una adecuada comunicación y coordinación entre los distintos niveles, que exista una dirección que guíe el cambio de situaciones, tome decisiones mediante un proceso continuo sistemático de análisis, que permita orientar los recursos disponibles hacia el logro de los objetivos de la organización.
Adicionalmente, se debe dar una observación continua tanto del ambiente interno como del medio externo de la organización; identificando fortalezas, debilidades, visualizando nuevas oportunidades o amenazas; enfocando tanto la misión como la visión de la empresa, a través de una gestión innovadora de dirección y liderazgo. Administrando efectivamente los recursos, todas estas tareas harán que se logren los objetivos, se den resultados esperados. Todo esto permitirá formular, implementar y evaluar la gestión de mantenimiento de la organización, para luego poder implementar el plan estratégico.

En esta perspectiva, para Duffuaa y otros (2010), la planificación del mantenimiento consiste en determinar los recursos necesarios para satisfacer la demanda de trabajos de mantenimiento. Estos recursos están relacionados con los equipos, herramientas, materiales, insumos, cantidad y competencias de la mano de obra. Además, están referidos a todo lo relacionado con el ambiente interno y externo de la organización. Estos autores señalan que, en la planificación de la gestión mantenimiento se deben seguir determinados pasos, para lograr una gestión eficiente, los cuales resumen en: plan estratégico, implementación del plan, ejecución del plan de mantenimiento, y su evaluación y control.

En palabras de Araujo (2008), la planificación del mantenimiento, es un proceso que se refiere a la existencia de una estructura organizada de planes de mantenimiento que estén alineados con las reales necesidades de los equipos; en casos en los que la cantidad de equipos sea importante, es necesario efectuar un análisis de criticidad. Otro autor consultado con respecto a este punto es Torres (2006), quien dice que la misma consiste en definir los objetivos que se quiere cumplir con la gestión de mantenimiento, basándose en la situación actual y los recursos de que se disponen; en este sentido, se fijan los objetivos y se avanza al mismo tiempo que se aseguran cada uno de ellos. 
Coincidiendo con los señalamientos antes expuestos, para la investigadora, planificar es decidir con anticipación el cómo hacer, el qué hacer, cuándo hacerlo, y quién debe hacerlo, con el fin de contribuir al logro de los objetivos de la empresa, considerando tanto su visión como estrategias a seguir. Es la base para llevar a cabo las acciones de mantenimiento, sin importar de que tipo sea el mismo, mejorando y teniendo de una manera ordenada los pasos a seguir, para que se cumpla el trabajo en sinergia.

Asimismo, con base a estos aspectos, la investigadora consideró pertinente el abordaje de la planificación en la gestión de mantenimiento a través del análisis de los pasos documentados por Difusa y otros (2010), en resumen: plan estratégico, implementación del plan, ejecución del plan de mantenimiento, y su evaluación y control.

\section{Plan estratégico}

Para Jiménez y Milano (2006, p. 42), el plan estratégico "es la definición teórica del que hacer, del futuro deseable de las organizaciones de mantenimiento para orientar los esfuerzos, el uso de los recursos y la relación con el entorno tanto a mediano como a largo plazo". El análisis del ambiente interno y externo de toda organización permite la formulación del plan estratégico, luego de haber seleccionado las estrategias adecuadas, es decir, la implementación del mismo. Permitiendo poner en marcha las estrategias con sus respectivos sistemas de evaluación o control. De hecho, Jiménez y Milano (2006), plantean que, en la formulación del plan estratégico deben existir pasos a seguir, entre estos identificar la misión, visión, objetivos y estrategias actuales.

Aunque, estos pasos no hayan sido planteados, comunicados o escritos formalmente, toda organización cuenta con estos elementos. Los mismos sirven para direccionar la evaluación interna y externa, además de ser un punto referencial para establecer nuevos objetivos, estrategias, misión y visión, en caso de ser necesaria. Por ello, dicha valoración se realiza con el objeto de identificar fortalezas, debilidades, oportunidades y amenazas. El diagnóstico de la situación de una organización de mantenimiento, exige la evaluación exhaustiva de una amplia variedad de factores relacionados.

Además, afirman Jiménez y Milano (2006), fijar la nueva misión del departamento de mantenimiento, donde debe quedar plasmado el propósito más importante, los cuales deben ser los miembros que la conforman, siendo esta su razón de ser y es considerada esencial para determinar tanto los objetivos como formular las estrategias. La selección de estrategias permitirá alcanzar los objetivos estratégicos, éstas representan las acciones que deben ser realizadas para lograr alcanzarlos.

En la práctica, continúan afirmando Jiménez y Milano (2006), las organizaciones generalmente fijan los objetivos y estrategias simultáneamente para periodos de 2 a 5 años, esto requiere visualizar tanto las fortalezas como debilidades, para después compararlas con las oportunidades y amenazas. Luego vincularlas con las distintas actividades dentro de la organización de mantenimiento que agregan valor. Por último, definir las políticas de la organización de mantenimiento, que según estos autores son líneas directrices específicas, los métodos, procedimientos, reglas que se establecen para implementar las estrategias que llevarán a alcanzar los objetivos enunciados.

Coincidiendo con estos planteamiento, en la Norma Venezolana COVENIN 2500 (1993) se expresa que, las organizaciones deben definir por escrito las estrategias, objetivos, metas y políticas que debe cumplir el departamento de mantenimiento, contentivo en un documento denominado plan estratégico, donde se especifiquen las necesidades reales y objetivas de mantenimiento para los diferentes objetos a mantener, establecer un orden de 
prioridades para la ejecución de acciones de mantenimiento de aquellos sistemas que lo requieren, además de ejecutar acciones de mantenimiento que se orienten hacia el logro de objetivos.

En este punto, la investigadora evidencia que, el plan estratégico dentro de la gestión de mantenimiento, es la definición teórica del que hacer, se realiza a través de la jerarquización de propósitos, objetivos, estrategias, políticas, procedimientos, reglas, programas, presupuestos, entre otros. Su objetivo primordial debe ser lograr con el mínimo coste, el mayor tiempo en servicio de los recursos que intervienen en el proceso productivo de la empresa, con el fin de conseguir la máxima disponibilidad, aportando la mayor productividad y seguridad de funcionamiento

\section{Implementación del plan estratégico}

Para Jiménez y Milano (2006), existen pasos claves a seguir en la implementación del plan estratégico. Mediante el plan operativo se debe elaborar el plan de mantenimiento para un año, definiendo los objetos de mantenimiento como serían equipos, sistemas, instalaciones, edificaciones, entre otros; asimismo, las actividades de mantenimiento, procedimientos de trabajo, programación de los mismos según el tipo de mantenimiento y el presupuesto anual. Además, señalan que se deben fijar metas a corto plazo en relación al plan anual de mantenimiento.

Al respecto de las metas, Jiménez y Milano (2006, p. 85) consideran que, "las metas se refieren a los resultados a corto plazo, las metas son esenciales para la ejecución acertada de las estrategias, ya que forman la base para la asignación de recursos y constituyen un elemento importante para controlar el avance hacia el logro de los objetivos estratégicos". Finalmente, asignar recursos, para poder enfrentar los retos del día a día de una organización de mantenimiento se precisa que la estrategia sea sucedida por la acción, es decir, llevar a la práctica la programación establecida en el plan de mantenimiento.

Ahora bien, Duffuaa y otros (2010) exponen que, en la identificación estratégica de recursos se deben tener presente tanto los recursos tangibles como los intangibles, humanos, materiales, organizacionales y financieros. Los recursos son usualmente identificados en términos de las fortalezas y debilidades de las empresas. Determinar lo que el departamento de mantenimiento debe hacer, solo es posible luego de establecer lo que éste puede hacer basándose en la calidad de sus recursos.

En este sentido, afirman estos autores, se deben identificar las fortalezas y debilidades en todos los niveles gerenciales, de forma tal que la alta gerencia pueda tener una visión global más clara de la posición de la empresa con relación al ambiente externo, mientras que los niveles más bajos de la dirección pueden tener una mejor comprensión de las fortalezas y debilidades internas.

Desafortunadamente, muchos administradores no piensan en términos de fortalezas y debilidades, como resultado, ellos se preocupan más sobre lo que deben hacer en lugar de preocuparse por lo que podrían hacer. Las grandes asociaciones cuentan con una gran cantidad de recursos ya sean financieros, tecnológicos, personal calificado, entre otros, pero por lo general reaccionan lentamente cuando los cambios así lo requieren.

Por otro lado, continúan argumentando los autores, las pequeñas empresas pueden reaccionar más rápidamente, pero tienen fortalezas limitadas; los elementos que influyen en el ambiente interno de cualquier organización pueden cambiar a través del tiempo, por lo tanto tienen que ser monitoreadas continuamente. Estas fortalezas y debilidades son una medida interna de lo que una agrupación puede hacer, deben estimarse basándose en la calidad de los recursos con los que cuenta la misma. 
Tal como lo señalan Duffuaa y otros (2010), los recursos tangibles: las fortalezas y debilidades de una organización son descritas usualmente en términos de sus recursos tangibles, la clasificación más común para estos recursos es la siguiente: recursos humanos, recursos materiales, recursos organizacionales, recursos financieros, recursos de información y tecnología.

En el área específica del mantenimiento el recurso humano puede clasificarse según el área técnica en la que se desempeñe, según García (2009): mecánica, electricidad, instrumentación, construcción, entre otros; es primordial la adecuada formación del personal de mantenimiento para contar con una fuerza laboral capaz y experimentada a fin de obtener un trabajo de mantenimiento de calidad, y en consecuencia lograr el éxito de la organización.

Por otra parte, acota García (2009), la labor del departamento de mantenimiento está relacionada, muy estrechamente, en la prevención de accidentes y lesiones en el trabajador ya que tiene la responsabilidad de mantener en buenas condiciones la maquinaria y herramienta, equipo de trabajo, lo cual permite un mejor desenvolvimiento y seguridad evitando en parte riesgos en el área laboral.

De igual manera, Jiménez y Milano (2006) consideran que, las empresas con abundantes recursos desde el punto de vista material, poseen una gran ventaja ante sus competidores y solo son superados por aquellas empresas que posean más recursos que ellos. Por otra parte, según Duffuaa y otros (2010), una empresa con un sólido recurso humano podría no poder tomar ventaja de las oportunidades a menos que tenga también los recursos físicos, es decir maquinarias, equipos, sistemas, entre otros, que se lo permitan. Las organizaciones de mantenimiento deben asegurar el suministro de todos los repuestos, herramientas, consumibles, suministros especiales necesarios para apoyar las tareas de mantenimiento.
Con base a lo analizado, la investigadora considera que, la implementación del plan estratégico conlleva al cumplimiento de ciertos pasos claves a seguir. La base para la efectiva implementación de dicho plan es la elaboración de los planes operativos, donde deben definirse los objetos a mantener, actividades de mantenimiento, procedimientos de trabajo, programación de los mismos, según el tipo de mantenimiento aplicable, y el presupuesto anual.

\section{Ejecución del plan estratégico}

Para la ejecución del plan de mantenimiento, las responsabilidades y actividades deben ser asignadas clara $y$ detalladamente por escrito a cada miembro de la organización de mantenimiento. Al respecto, para Jiménez y Milano (2006), algunas responsabilidades que pueden ser usadas dentro de una organización de mantenimiento, son:

$\checkmark$ Planificar y programar eficientemente la ejecución de los trabajos, a fin de reducir las paradas, asegurar calidad de las reparaciones, y garantizar la adecuada distribución y asignación eficiente de los recursos.

$\checkmark$ Adiestrar efectiva y eficientemente el recurso humano, revisando continuamente los procedimientos a fin de mejorar su eficiencia.

$\checkmark$ Revisar continuamente el grado de obsolescencia de los equipos para programar los reemplazos de forma oportuna y económica.

$\checkmark$ Velar por el estricto cumplimiento de las normas de protección integral y crear en los trabajadores una fuerte conciencia sobre la necesidad de acatarlas.

$\checkmark$ Distribuir y asignar los recursos de acuerdo con la criticidad y riesgos de los equipos.

$\checkmark$ Desarrollar la ingeniería de mantenimiento, para que sea el soporte técnico. 
Coincidiendo con estos autores, Zambrano y Leal (2006) plantean que, la ejecución del plan de mantenimiento es la etapa del proceso de gestión donde se realizan las actividades previamente identificadas, planificadas y programadas. Comprende, por tanto, la realización de las actividades relativas a la ejecución de mantenimiento tanto el rutinario como el programado, así como el chequeo de mantenimiento rutinario y circunstancial. Una vez completado el trabajo, es esencial recibir información sobre los resultados para medir la eficacia del proceso y lograr una mayor eficiencia a futuro.

En virtud de lo expuesto, para la investigadora, la ejecución del plan consiste en la realización de las actividades de mantenimiento, referidas a la aplicación de los distintos tipos de según los requerimientos identificados, planificados y programados. En esta etapa, se genera la información sobre los resultados, lo cual servirá para medir la eficacia global del proceso y lograr una mayor eficiencia en el futuro.

\section{Evaluación y control del plan estratégico}

De acuerdo a Jiménez y Milano (2006), la evaluación y control se realiza en dos niveles. Primeramente, se evalúa y controla el plan anual de mantenimiento mediante los indicadores definitivos, establecidos previamente en la etapa de implementación del plan estratégico, los cuales permitirán medir las desviaciones más relevantes entre los resultados reales y los programados como metas, a fin de retroalimentar el proceso con los correctivos necesarios a corto plazo, seguidamente se evalúa el plan estratégico para retroalimentar el proceso reafirmando las estrategias o sugiriendo cambios.

El enfoque tradicional ha consistido en destacar las etapas como pasos secuenciales en la planificación estratégica. Esto es lo que se conoce como estrategias planificadas. Sin embargo, desde el interior de la organización pueden surgir, sin planificación previa, estrategias llamadas emergentes sin seguir la secuencia anterior (Jiménez y Milano, 2006). Esto debe ser evaluado comparando las estrategias emergentes con la misión y la visión, así como también con las fortalezas, oportunidades, debilidades y amenazas. El objetivo consiste en, evaluar si las estrategias emergentes se adecuan tanto a las necesidades como a las capacidades organizacionales.

Una de las formas más efectivas, para realizar seguimiento del avance y medir la efectividad del plan estratégico de mantenimiento, a juicio de Gómez y Cote (2011), es realizar periódicamente (al menos una vez al año) la evaluación de la gestión de mantenimiento, de esta forma se podrán tomar acciones para corregir desviaciones en los resultados esperados, así enfocar los esfuerzos en la obtención de estos resultados. La evaluación del plan estratégico de mantenimiento, se debe realizar a través de una serie de indicadores que permitirán conocer su cumplimiento, además de la efectividad del mismo, que se deberá ver reflejado en un mejoramiento de los indicadores claves de desempeño.

A efectos de la investigación, en la evaluación y control del plan estratégico, se debe evaluar y controlar el plan anual de mantenimiento mediante los indicadores definitivos, lo cual permitirá evaluar el plan estratégico para retroalimentar el proceso reafirmando las estrategias o sugiriendo cambios. En este aspecto, se acota que, la evaluación del plan estratégico de mantenimiento debe realizarse a través de una serie de indicadores que permitirán conocer su cumplimiento, además de la efectividad del mismo, que se deberá ver reflejado en un mejoramiento de los indicadores claves de desempeño. 


\section{MÉTODO}

El tipo de investigación se contextualizo como descriptivo con un diseño no experimental, transeccional y de campo. La población de estudio quedó constituida por 22 personas que se desempeñan en el área de mantenimiento de las universidades públicas de la Costa Oriental del Lago: Universidad del Zulia, Núcleo Costa Oriental del Lago, Universidad Nacional Experimental "Rafael María Baralt" y, finalmente, la Universidad Politécnica Territorial del Zulia, sede Cabimas.

La técnica para la recolección de datos fue la encuesta, siendo el instrumento un cuestionario conformado por 12 ítems con cinco alternativas de respuestas dadas por las siguientes alternativas: siempre, casi siempre, algunas veces, casi nunca y nunca, el mismo fue validado por cinco expertos, mientras que su confiabilidad se determinó a través del coeficiente de Alpha Cronbach, cuyo resultado fue de 0,932.

Una vez obtenida la información relacionada con el instrumento de recolección, se procedió a su codificación y tabulación por ítem, haciendo uso de la estadística descriptiva a través de la media aritmética por ítem e indicador, mostrándose de esta forma la opinión emitida por los sujetos que conforman la población. Es importante resaltar que para interpretar la media aritmética se construyó un baremo para el análisis de la media aritmética, tal como se muestra en la Tabla 1.

Tabla 1. Baremo para la interpretación de la media aritmética

\begin{tabular}{|c|c|c|}
\hline INTERVALO & CATEGORÍA & INTERPRETACIÓN \\
\hline $4.21-5.00$ & Muy alta presencia & $\begin{array}{l}\text { Muy alta presencia del ítem, indicador, y dimensión. } \\
\text { Implica muy alta fortaleza para las universidades } \\
\text { publicas }\end{array}$ \\
\hline $3.41-4.20$ & Alta presencia & $\begin{array}{l}\text { Alta presencia del ítem, indicador, y dimensión. } \\
\text { Implica alta fortaleza para las universidades publicas }\end{array}$ \\
\hline $2.61-3.40$ & Moderada presencia & $\begin{array}{l}\text { Moderada presencia del ítem, indicador, y dimensión. } \\
\text { Implica leve fortaleza para las universidades publicas }\end{array}$ \\
\hline $1.81-2.60$ & Baja presencia & $\begin{array}{l}\text { Baja presencia del ítem, indicador, y dimensión. } \\
\text { Implica alta debilidad para las universidades publicas }\end{array}$ \\
\hline $1.00-1.80$ & Muy baja presencia & $\begin{array}{l}\text { Muy baja presencia del ítem, indicador, y dimensión. } \\
\text { Implica muy alta debilidad para las universidades } \\
\text { publicas }\end{array}$ \\
\hline
\end{tabular}

Fuente: Elaboración propia (2019)

\section{RESULTADOS}

A efectos de iniciar el análisis, se presentan los resultados obtenidos para cada uno de los indicadores que conforman la dimensión planificación del mantenimiento, y se dividió en cuatro (4) indicadores: plan estratégico, implementación del plan estratégico, ejecución del plan estratégico y la evaluación y control del plan estratégico.
En la tabla 2 se presenta el resumen para el análisis estadístico del indicador plan estratégico, observándose una media aritmética para el indicador de 4,33 lo cual indica que el plan estratégico como parte de la planificación del mantenimiento, arriba a una categoría de muy alta presencia, según el baremo diseñado, implicando muy alta fortaleza. 
Tabla 2. Indicador: Plan estratégico

\begin{tabular}{|c|c|c|c|}
\hline \multicolumn{3}{|c|}{ Planificación del mantenimiento } & \\
\hline \multicolumn{3}{|c|}{ En la universidad pública donde usted labora: } & CATEGORÍA \\
\hline 1 & $\begin{array}{l}\text { Actualizan el plan estratégico de mantenimiento } \\
\text { considerando los cambios que se suscitan en el entorno } \\
\text { organizacional. }\end{array}$ & 3,64 & $\begin{array}{l}\text { Alta presencia } \\
\text { Alta fortaleza }\end{array}$ \\
\hline & Formulan objetivos en el plan estratégico de mantenimiento & & Muy alta presencia \\
\hline 2 & que contribuyan al logro de los objetivos organizacionales. & 4,55 & Muy alta fortaleza \\
\hline & Analizan el ambiente interno de la gerencia antes de & & Muy alta presencia \\
\hline 3 & elaborar el plan estratégico. & 4,81 & Muy alta fortaleza \\
\hline \multirow{2}{*}{\multicolumn{2}{|c|}{ Total indicador: }} & 4,33 & Muy alta presencia \\
\hline & & 4,00 & Muy alta fortaleza \\
\hline
\end{tabular}

Fuente: Elaboración propia (2019)

Al analizar detalladamente los resultados obtenidos para cada uno de los ítems del indicador, pudo visualizarse en relación a los ítems 2 y 3 muy alta presencia de estas actividades, implicando que en estas universidades se realiza con muy alta frecuencia lo referente a: formulan objetivos en el plan estratégico de mantenimiento que contribuyan al logro de los objetivos organizacionales, evidenciando una media de 4,55; y analizan el ambiente interno de la gerencia antes de elaborar el plan estratégico $(4,81)$; adjudicándole muy altas fortalezas a la gestión de mantenimiento realizada.

Al mismo tiempo, se evidencia alta presencia de que actualicen el plan estratégico de mantenimiento considerando los cambios que se suscitan en el entorno organizacional $(3,64)$, implicando una alta fortaleza al respecto.

Considerando que el indicador arriba a la categoría de muy alta presencia, se puede decir que se validan los postulados teóricos de Jiménez y Milano (2006), para quienes el plan estratégico es la definición teórica del que hacer, del futuro deseable de las organizaciones de mantenimiento para orientar los esfuerzos, el uso de los recursos y la relación con el entorno tanto a mediano como a largo plazo.

Asimismo, son congruentes con la posición de la investigadora, para quien el plan estratégico en la gestión de mantenimiento, es la definición teórica del que hacer, se realiza a través de la jerarquización de propósitos, objetivos, estrategias, políticas, procedimientos, reglas, programas, presupuestos, entre otros. Su objetivo primordial debe ser lograr con el mínimo coste, el mayor tiempo en servicio de los recursos que intervienen en el proceso productivo de la empresa, con el fin de conseguir la máxima disponibilidad, aportando la mayor productividad y seguridad de funcionamiento.

Seguidamente, en la tabla 3, se muestran los resultados obtenidos del indicador implementación del plan estratégico, perteneciente a la dimensión que se analiza. Se observa una media para el indicador de 3,32, indicando que la implementación del plan estratégico como parte de la planificación del mantenimiento, ostenta una categoría de moderada presencia, otorgándole a esta gestión una leve fortaleza. 
Tabla 3. Indicador: Implementación del plan estratégico

\begin{tabular}{|c|c|c|c|}
\hline \multicolumn{4}{|c|}{ Planificación del mantenimiento } \\
\hline & ÍTEMS & & \\
\hline \multicolumn{2}{|c|}{ En la universidad pública donde usted labora: } & $\overline{\boldsymbol{X}}$ & CATEGORÍA \\
\hline \multirow{3}{*}{4} & Establecen cronograma de ejecución de las labores de & & Moderada presencia \\
\hline & mantenimiento. & 3,32 & Leve fortaleza \\
\hline & Cuentan con los recursos organizacionales necesarios & & Moderada presencia \\
\hline \multirow[t]{2}{*}{5} & para implementar el plan de mantenimiento. & 2,62 & Leve fortaleza \\
\hline & Asignan oportunamente los recursos necesarios para & & Alta presencia \\
\hline \multirow[t]{2}{*}{6} & ejecutar el plan de estratégico de mantenimiento. & 4,02 & Alta fortaleza \\
\hline & & & Moderada presencia \\
\hline \multicolumn{2}{|c|}{ Total indicador: } & 3,32 & Leve fortaleza \\
\hline
\end{tabular}

Fuente: Elaboración propia (2019)

Al detallar los resultados para cada ítem, se observa que solo el ítem 6 arriba a la categoría de alta presencia, donde se les consulta si asignan oportunamente los recursos necesarios para ejecutar el plan de estratégico de mantenimiento $(4,02)$, siendo esto alta fortaleza de la gestión. No obstante, otorgaron moderada presencia al resto de los reactivos, indicando una moderada frecuencia del que: establezcan cronograma de ejecución de las labores de mantenimiento $(3,32)$, y cuenten con los recursos organizacionales necesarios para implementar el plan de mantenimiento $(2,62)$; signando estas actividades como leves fortalezas de la gestión analizada.

Las afirmaciones precedentes validan medianamente a Jiménez y Milano (2006), cuando afirman que, existen pasos claves a seguir en la implementación del plan estratégico. Mediante el plan operativo se debe elaborar el plan de mantenimiento para un año, definiendo los objetos de mantenimiento como serían equipos, sistemas, instalaciones, edificaciones, entre otros; asimismo, las actividades de mantenimiento, procedimientos de trabajo, programación de los mismos según el tipo de mantenimiento y el presupuesto anual.

De igual manera, se alcanza mediana coincidencia con lo expuesto por la investigadora, quien considera que, la implementación del plan estratégico conlleva al cumplimiento de ciertos pasos claves a seguir. La base para la efectiva implementación de dicho plan es la elaboración de los planes operativos, donde deben definirse los objetos a mantener, actividades de mantenimiento, procedimientos de trabajo, programación de los mismos, según el tipo de mantenimiento aplicable, y el presupuesto anual.

De seguido, en la tabla 4, se muestran los resultados obtenidos para el indicador ejecución del plan estratégico, perteneciente a la dimensión planificación del mantenimiento, donde se observa que la media se posicionó en 3,61, indicando alta presencia de la ejecución del plan estratégico en la planificación del mantenimiento de las universidades estudiadas, confiriéndoles, por tanto, una alta fortaleza a su gestión. 
Tabla 4. Indicador: Ejecución del plan estratégico

\begin{tabular}{llll}
\hline \multicolumn{5}{c}{ Planificación del mantenimiento } & & \\
En la universidad pública donde usted labora: & $\overline{\boldsymbol{X}}$ & CATEGORÍA \\
\hline $7 \quad \begin{array}{l}\text { Planifican la ejecución de los trabajos de } \\
\text { mantenimiento a fin de asegurar la calidad de las } \\
\text { reparaciones. }\end{array}$ & 3,55 & $\begin{array}{l}\text { Alta presencia } \\
\text { Alta fortaleza }\end{array}$ \\
$\begin{array}{l}\text { Velan por el estricto cumplimiento de las normas } \\
\text { durante la ejecución del mantenimiento. }\end{array}$ & 2,79 & $\begin{array}{l}\text { Moderada presencia } \\
\text { Leve fortaleza }\end{array}$ \\
$\begin{array}{l}\text { Asignan los recursos organizacionales de acuerdo con } \\
\text { la criticidad de los activos a los cuales se ejecutarán } \\
\text { labores de mantenimiento. }\end{array}$ & 4,49 & $\begin{array}{l}\text { Muy alta presencia } \\
\text { Muy alta fortaleza }\end{array}$ \\
Total indicador: & 3,61 & $\begin{array}{l}\text { Alta presencia } \\
\text { Alta fortaleza }\end{array}$ \\
\hline
\end{tabular}

Fuente: Elaboración propia (2019)

Como se puede apreciar, se otorga muy alta presencia a lo relacionado a si, asignan los recursos organizacionales de acuerdo con la criticidad de los activos a los cuales se ejecutarán labores de mantenimiento $(4,49)$, implicando una muy alta fortaleza en este sentido. Al mismo tiempo, se evidencia alta presencia, por ende alta fortaleza, de que planifiquen la ejecución de los trabajos de mantenimiento a fin de asegurar la calidad de las reparaciones $(3,55)$. Sin embargo, calificaron con moderada presencia el que velen por el estricto cumplimiento de las normas durante la ejecución del mantenimiento $(2,79)$, representando esto una leve fortaleza.

Estos resultados permiten afirmar alta coincidencia con lo planteado por Zambrano y Leal (2006) quienes plantean que, para la ejecución del plan de mantenimiento, las responsabilidades y actividades deben ser asignadas clara y detalladamente por escrito a cada miembro de la organización de mantenimiento.

De igual manera, se valida la posición de la investigadora, para quien la ejecución del plan de mantenimiento consiste en la realización de las actividades relativas a la ejecución de mantenimiento, referidas a la aplicación de los distintos tipos de mantenimiento según los requerimientos identificados, planificados y programados. En esta etapa, se genera la información sobre los resultados, lo cual servirá para medir la eficacia global del proceso y lograr una mayor eficiencia en el futuro.

De acuerdo a los valores de la tabla 5, del indicador evaluación y control del plan estratégico, se tiene una media de 4,12 indicando alta presencia del indicador, según lo establecido en el baremo, implicando alta fortaleza para la gestión de mantenimiento en este aspecto. Desde el punto de vista de los resultados por ítem, en estas universidades se visualiza muy alta presencia en lo concerniente a si implementan oportunamente acciones correctivas al momento de detectar desviaciones en los planes estratégicos $(4,12)$, indicando esto como muy alta fortaleza de la gestión. 
Tabla 5. Indicador: Evaluación y control del plan estratégico

\begin{tabular}{|c|c|c|c|}
\hline \multicolumn{4}{|c|}{$\begin{array}{l}\text { Planificación del mantenimiento } \\
\text { ÍTEMS }\end{array}$} \\
\hline & ia de operaciones acuática donde usted labora: & $X$ & CAIEGORIA \\
\hline 10 & $\begin{array}{l}\text { Evalúan el plan anual de mantenimiento mediante } \\
\text { indicadores de gestión. }\end{array}$ & 3,62 & $\begin{array}{l}\text { Alta presencia } \\
\text { Alta fortaleza }\end{array}$ \\
\hline & Evalúan de forma periódica la gestión del & & Alta presencia \\
\hline 11 & mantenimiento. & 3,94 & Alta fortaleza \\
\hline 12 & $\begin{array}{l}\text { Implementan oportunamente acciones correctivas al } \\
\text { momento de detectar desviaciones en los planes } \\
\text { estratégicos. }\end{array}$ & 4,81 & $\begin{array}{l}\text { Muy alta presencia } \\
\text { Muy alta fortaleza }\end{array}$ \\
\hline \multicolumn{2}{|c|}{ Total indicador: } & 4,12 & $\begin{array}{l}\text { Alta presencia } \\
\text { Alta fortaleza }\end{array}$ \\
\hline
\end{tabular}

Fuente: Elaboración propia (2019)

Mientras, consideraron como de alta presencia lo referido a si evalúan el plan anual de mantenimiento mediante indicadores de gestión $(3,62)$ ítem 10 , y evalúan de forma periódica la gestión del mantenimiento $(3,94)$ ítem 11; confiriéndoles altas fortalezas en estos aspectos.

Las evidencias anteriores, de alta presencia, se apegan a los postulados teóricos de González (2004), para quien la evaluación del plan estratégico de mantenimiento, se debe realizar a través de una serie de indicadores que permitirán conocer su cumplimiento, además de la efectividad del mismo, que se deberá ver reflejado en una mejoramiento de los indicadores claves de desempeño.

De la misma manera, estas evidencias validan la posición de la investigadora cuando afirma que, en la evaluación y control del plan estratégico, se debe evaluar y controlar el plan anual de mantenimiento mediante los indicadores definitivos, lo cual permitirá evaluar el plan estratégico para retroalimentar el proceso reafirmando las estrategias o sugiriendo cambios.

Seguidamente, en la tabla 6 se resume el comportamiento de la dimensión planificación del mantenimiento, la cual fue medida por sus indicadores plan estratégico, implementación del plan estratégico, ejecución del plan estratégico y la evaluación y control del plan estratégico. Se observa que esta planificación fue catalogada en la categoría de alta presencia, con una media alcanzada de 3,85, confiriéndole a esta gestión de mantenimiento una alta fortaleza en lo que concierne a esta dimensión.

Tabla 6. Dimensión: Planificación en la gestión de mantenimiento

\begin{tabular}{lcl}
\hline & Planificación del mantenimiento \\
INDICADORES & $\overline{\boldsymbol{X}}$ & CATEGORÍAS \\
\hline Plan estratégico & 4,33 & Muy alta presencia/Muy alta fortaleza \\
Implementación del plan estratégico & 3,32 & Moderada presencia /Leve fortaleza \\
Ejecución del plan estratégico & 3,61 & Alta presencia/Alta fortaleza \\
Evaluación y control del plan estratégico & 4,12 & Alta presencia/Alta fortaleza \\
RESUMEN DIMENSIÓN & 3,85 & Alta presencia/Alta fortaleza \\
\hline
\end{tabular}

Fuente: Elaboración propia (2019) 
Asimismo, se evidencia que el plan estratégico arriba a muy alta presencia con una media aritmética de 4,33, siendo esto muy alta fortaleza de la gestión; a su vez, la ejecución del plan estratégico así como la evaluación y control del plan estratégico muestran alta presencia, confiriéndoles a las universidades estudiadas altas fortalezas en estos aspectos; no obstante, la implementación del plan estratégico se ubicó con la categoría de moderada presencia, representando leve fortaleza de la gestión, evidenciando oportunidades de mejora en este sentido.

Estos señalamientos verifican, en alta medida, los postulados de Duffuaa y otros (2010), para quienes la planificación del mantenimiento consiste en determinar los recursos necesarios para satisfacer la demanda de trabajos de mantenimiento. Estos recursos están relacionados con los equipos, herramientas, materiales, insumos, cantidad y competencias de la mano de obra. Además, están referidos a todo lo relacionado con el ambiente interno y externo de la organización.

De igual manera, se valida lo planteado por estos autores cuando señalan que, en la planificación del mantenimiento se deben seguir determinados pasos, para lograr una gestión eficiente, los cuales se resumen en: plan estratégico, implementación del plan, ejecución del plan de mantenimiento, y su evaluación y control.

Asimismo, se logra una alta coincidencia con la construcción teórica de la investigadora cuando señala que, planificar es decidir con anticipación el cómo hacer, el qué hacer, cuándo hacerlo, y quién debe hacerlo, con el fin de contribuir al logro de los objetivos de la empresa, considerando tanto su visión como estrategias a seguir. Es la base para llevar a cabo las acciones de mantenimiento, sin importar de que tipo sea el mismo, mejorando y teniendo de una manera ordenada los pasos a seguir, para que se cumpla el trabajo en sinergia.

\section{CONCLUSIONES}

En relación al objetivo, donde se describió la planificación del mantenimiento en las universidades públicas de la Costa Oriental del Lago, se concluye que estas organizaciones otorgan alta presencia a este proceso de planificación en su gestión de mantenimiento, lo cual les atribuye una alta fortaleza.

Sin embargo, se acota que aun con esta categoría de alta presencia alcanzada, se detectó la existencia de ciertos aspectos a ser reforzados, específicamente en cuanto a la implementación y ejecución del plan estratégico los cuales mostraron actividades catalogadas con moderada presencia. Estos aspectos muestran oportunidades de mejora, motivo por el cual las universidades debe revisarlos, en virtud de la importancia que tienen en la gestión de mantenimiento, debido a su efecto en toda la organización; de ello dependerá estrechamente el desarrollo de la gestión, a través del logro de sus objetivos.

\section{REFERENCIAS BIBLIOGRÁFICAS}

Alpizar, R. (2005). Gestión de Mantenimiento. McGraw-Hill Interamericana. Colombia.

Amendola, L. (2006). Gestión de proyectos de activos industriales. Editorial Universidad Politécnica de Valencia. Valencia. España

Arata, A. (2009). Ingeniería y gestión de la confiabilidad operacional en plantas industriales. Ril Editores. Chile

Araujo, J. (2008). Gestión del mantenimiento. Editorial el Búho. Bogotá, Colombia.

Dounce, E. (2011). El mantenimiento industrial. Revista Mantenimiento en Latinoamérica. La revista para la gestión confiable de activos. Vol. 3 № 2. MarzoAbril 2011. Bolivia. Disponible en: http://www. pistarelli.com.ar/MLV32.pdf

Duffuaa, S., Raouf, A. y Campbell, J. (2010). Sistemas de Mantenimiento. Planificación y Control. Editorial Limusa. México

García, S. (2009). Organización y gestión integral de mantenimiento. Manual práctico para la implantación de sistemas de gestión avanzados de mantenimiento 
industrial. Ediciones Díaz de Santos, S.A. Madrid - España

Gómez, E. y Cote, D. (2011). La productividad en el mantenimiento industrial. 2da Edición. Editorial. CECSA. México D.F

Jiménez, K. y Milano, T. (2006). Planificación y gestión del mantenimiento industrial. Un enfoque estratégico y operativo. Editorial Panapo. Caracas. Venezuela

Kelly, A. (2006). Strategic Maintenance Planning. Elsevier Ltd. Oxford. USA

Koontz, H. y Weihrich, H. (2008). Elementos de la administración. Enfoque internacional 6ta Edición. Editorial McGraw-Hill Interamericana Editores, S.A. México

Matalobos, J. (2003). Manual del ingeniero industrial. Editorial McGraw-Hill. México.

Nava, E. (2006). Fundamentos básicos del mantenimiento industrial. Grupo Editorial Norma. Bogotá. Colombia

Norma COVENIN 2500 (1993). Manual para evaluar los sistemas de mantenimiento en la industria. 1ra. Edición. Comisión Venezolana de Normas Industriales. Publicación FONDONORMA. Caracas. Venezuela

Rodríguez, J. (2008). Gestión de mantenimiento. Introducción a la teoría del mantenimiento. Casos empresariales. Documento en línea. Disponible en http://www.scribd.com/doc/7497765/gesti on-del-mantenimiento

Torres, N. (2006). Como realizar mantenimiento. Editorial Norma. Caracas. Venezuela

Zambrano, E. (2008). Valores éticos y toma de decisiones de los gerentes de las universidades publicas venezolanas. Tesis Doctoral Universidad del Zulia. Venezuela

Zambrano, S. y Leal, S. (2006). Manual práctico de gestión de mantenimiento. Fondo Editorial UNET. San Cristóbal. Venezuela 